\title{
A blend of comforting strategies and a form of team comforting were used during nasogastric tube insertion
}

Penrod J, Morse JM, Wilson S. Comforting strategies used during nasogastric tube insertion.J Clin Nurs 1999 Jan;8:31-8.

\section{Question}

How do nurses use patient comforting strategies to facilitate the insertion of a nasogastric tube in a trauma care setting?

\section{Design \\ Qualitative ethology.}

\section{Setting}

3 level 1 trauma centres.

\section{Data source}

The investigators videotaped 32 trauma cases during which there were 49 attempts to insert a nasogastric tube. Patients were 2-74 years of age and most were men (71\%). Trauma team members who inserted the tube were nurses ( $55 \%$ of attempts), physicians $(37 \%)$, or students $(8 \%)$.

\section{Methods}

Data were analysed using content analysis, and detailed textual descriptions of behaviour patterns used to comfort patients were produced. Using an existing typology of comforting strategies, recurring behaviours were identified and categorised.

\section{Main findings}

Nurses selectively used comforting strategies that focused on the competing interests of successful tube insertion and minimisation of patient discomfort. Direct comforting strategies were the primary tools used to achieve patient comfort. Nurses began with an assessment that focused on the procedure, but also addressed more global aspects of patient experiences such as pain. During insertion, nurses became focused on the procedure and secondary team members took over global assessment. Nurses used comforting talk to maintain patient focus and cooperation. Speech was patterned and repetitious, and intona- tion was melodic and rhythmic (eg, “... Start swallowing. Big swallow, swallow it down...")

Indirect comforting strategies involved measures to create a relaxed environment in which patients felt safe. Keeping things cool during insertion was achieved by (1) the message or choice of words (eg, use of first names and describing the procedure as uncomfortable rather than terrible); (2) the delivery (eg, voices had a sing-song quality, speech was slower and at a normal to loud volume); and (3) image or body language (eg, calm and relaxed, with unhurried but efficient movements). Pacing refocused the team on the total patient experience rather than just the immediate procedure. Both nurses and physicians reminded team members of the intended progression of care and the patient's need for pain medication or rest between insertion attempts. Sometimes a team member would physically block another member to interrupt the pace of care (eg, nurse moved over the patient to wipe her face, helping the patient to refocus and forcing the physician to wait before another insertion attempt).

Strategies such as light melodic talk were often augmented with various modes of touching (eg, patting patient on arm). Nurses adjusted their use of comfort talk and touch in response to patient cues.

\section{Conclusions}

During nasogastric tube insertion in a trauma care setting, nurses balanced efforts to complete the procedure successfully with strategies to minimise patient discomfort. Individual team members and the collective team shifted their comforting behaviours in response to the needs of the procedure and patient and in response to the behaviours of other team members.

Source of funding: National Institute of Nursing Research.

For correspondence: $\operatorname{Dr} J$ M Morse, International Institute for Qualitative Methodology, University of Alberta, 8303-112 Street, Edmonton, AB T6G 2K4, Canada. Fax +1 7804929040.

\section{Commentary}

This study by Penrod et al explored the nature of caring during the insertion of a nasogastric tube in a trauma care setting. The most obvious goal of many trauma care procedures is to stabilise the patient's medical condition and ensure survival. However, in the fast paced, intense, context of providing trauma care, it is also important to address a patient's psychological needs-to provide reassurance and to allay fears and confusion. Not only will this help the patient to get through what is often a terrifying and painful experience but it also can promote patient trust and cooperation, enabling the trauma care team to efficiently and effectively complete the necessary medical procedures.

The authors used qualitative ethology, a systematic, detailed study of behaviour in context. Specifically, they reviewed videotapes of 49 attempts at nasogastric tube insertion in 3 level 1 trauma centres to identify the strategies used by caregivers to comfort patients. The authors have studied comforting interaction extensively and used their own typology of comforting as the basis for the analysis of the data from this study. A degree of caution needs to be exercised, however, when applying the findings of North American research to health care in other cultures. What may be appropriate caring behaviour in one society may not be appropriate in another.

How can the findings of this study be applied in practice? This study identified and described various strategies that were used by individual team members, and further sought to explicate the interplay between patient and caregiver, and between various members of the trauma team. Future research should be done to determine the effects of such strategies on patient anxiety and discomfort. The findings of this study can help nurses who administer invasive or uncomfortable procedures to become more aware of the comforting strategies that they use, and the roles that they can adopt to meet the medical and psychological needs of their patients.

Herrick R Dean, RN, RNT, BA
Nurse Analyst
April Cottage, Nailsea
Bristol, UK

